\title{
Precise computation for the response of ultrasonic transmission systems by numerical multiple integration
}

\author{
Tohru Imamura \\ National Research Laboratory of Metrology, \\ 1-1-4, Umezono, Sakura-mura, Niihari-gun, Ibaraki, 305 Japan
}

(Received 21 September 1985)

\begin{abstract}
Particle velocity of the system of a pair of square transducers and that of circular ones are computed precisely and are compared with each other. The principle for the computation is based on Rayleigh's theory, and numerical multiple integration is achieved. In the computation, repeated trapezoidal rule is used, and how the result converges with the increase of the number of subintervals is investigated. The convergency of the system of a pair of square transducers by numerical quadruple integration and that of circular transducers by numerical double integration with analytical decrease of integral multiplicity are revealed to be different. Particle velocity and velocity potential multiplied by $2 \pi / \lambda$ show almost the same convergency.
\end{abstract}

PACS number: 43. 20. $\mathrm{Bi}$

\section{INTRODUCTION}

Diffraction effects in ultrasonic field have long been investigated by many authors. ${ }^{1-9)}$ In the history, digital computers have proved to be effective for these purposes. ${ }^{4}$ Recently, the author achieved numerical quadruple integration for the analysis of ultrasonic transmission system of rectangular transducers. ${ }^{9)}$ To deepen these analyses, precise computation is achieved, and, how the integral converges with the increase of the number of subintervals is investigated, and the results are tabulated. The case of circular transducers is also analyzed and is compared with that of the square ones.

\section{RESPONSE OF ULTRASONIC TRANSMISSION SYSTEMS}

An ultrasonic transmission system is composed of a transmitter, medium of ultrasonic propagation and a receiver. This system transforms the signal on the transmitter to that on the receiver. The response of this system can be calculated theoretically by Rayleigh's equation which integrates the point source acoustic propagation equations for spherical waves over the transducer areas. In general, quadruple numerical integration is necessary, and, it is difficult to decrease analytically the integral multiplicity for the square transducers.

Sinusoidal particle velocity is assumed on the transmitter. The medium of the ultrasonic propagation is assumed to be isotropic and non-absorptive. As the response of the ultrasonic transmission systems, particle velocity is calculated.

Figure 1 shows the coordinate system where square transducers are used as a transmitter and a receiver. The particle velocity $\dot{v}$ at a point $\mathbf{P}\left(x^{\prime}, y^{\prime}, 0\right)$ on the transmitter is assumed to be expressed as

$$
\dot{v}\left(x^{\prime}, y^{\prime}, 0, t\right)=\dot{v}_{0} \exp (j \omega t),
$$

where $\dot{v}_{0}$ is the complex amplitude of the particle velocity, $t$ the time and $\omega$ the angular frequency. So, the velocity potential $\dot{\Phi}$ at a point $\mathrm{Q}(x, y, z)$ on the receiver yielded by the point source $\mathbf{P}$ is expressed as

$$
\begin{aligned}
& \dot{\Phi}_{\mathrm{PQ}}(x, y, z, t) \\
& \quad=(\dot{v} / 2 \pi d) \times \exp \{j(\omega t-k d)\},
\end{aligned}
$$

where $k$ is the wave number of the ultrasonic wave and $d$ the distance between $\mathrm{P}$ and $\mathrm{Q}$. Then, the nor- 


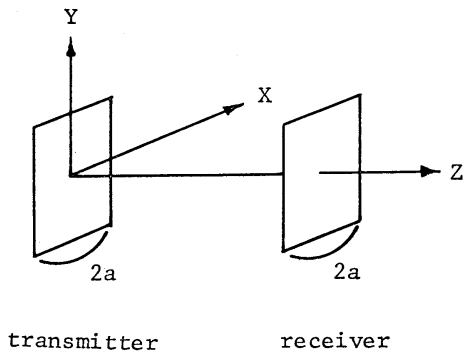

Fig. 1 Coordinate system used in the computation.

mal particle velocity at the point $\mathrm{Q}$ is given as

$$
\begin{aligned}
\dot{v}_{\mathrm{PQ}}= & -\partial \dot{\Phi}_{\mathrm{PQ}} / \partial z \\
= & \dot{v}_{0} \exp (j \omega t) z(j k d+1) / \\
& 2 \pi d^{3} \times \exp (-j k d),
\end{aligned}
$$

which can be expressed as

$$
\begin{aligned}
& \dot{v}_{\mathrm{PQ}}(x, y, z, t) \\
& \quad=\dot{v}_{0} \exp (j \omega t) R_{\mathrm{PQ}} \exp \left(-j \phi_{\mathrm{PQ}}\right),
\end{aligned}
$$

where $R_{\mathrm{PQ}}$ and $\phi_{\mathrm{PQ}}$ do not depend on time. These are the amplitude and the phase delay of the ultrasonic transmission system of a pair of point transducers, namely, the response of this system.

The response of the system of a line transducer and a point one is computed by integrating Eq. (3) over the line transducer, and can be expressed as Eq. (3)'. That of a square transducer and a point one is computed by integrating Eq. (3) over the square transducer, and can be expressed as before. That of a square transducer and a line one is computed by integrating Eq. (3) over the square transducer and a line one, and also can be expressed as before. That of a pair of square transducers are computed by integrating Eq. (3) over the two square transducers.

If the transducer is circular, the two dimensional integration on the transducer can be reduced to a single integration. Figure 2 shows how this is completed. In the figure, the following equations hold:

$$
\begin{gathered}
q \sin \theta=a \sin \theta_{0}, \\
q \cos \theta=r+a \cos \theta_{0} .
\end{gathered}
$$

By setting as

$$
u=\left(q^{2}+r^{2}-a^{2}\right) / 2 q r,
$$

$\theta$ can be expressed as

$$
\theta=\tan ^{-1}\left(\sqrt{1-u^{2}} / u\right), \quad(0 \leqq \theta \leqq \pi) .
$$

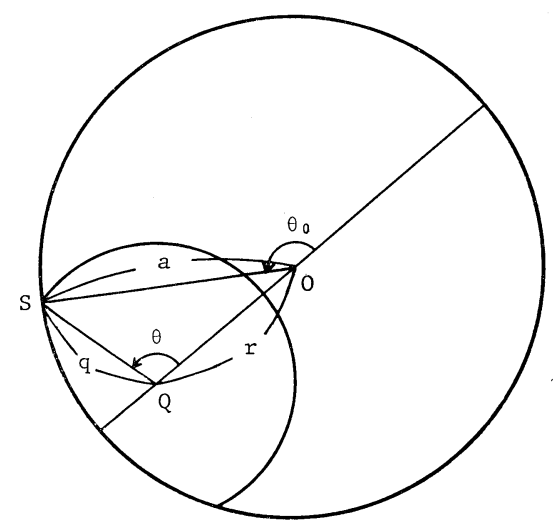

Fig. 2 Method to disintegrate analytically double integral to single one for a circular transducer.

When point $O$ is the central axis of the transmitter and point $Q$ is the place where particle velocity is computed, the contribution on the arc with center $\mathrm{Q}$ and radius $q$ is equal. The length of the $\operatorname{arc}$ can be expressed by the function of the radius of the transmitter $(a)$, the distance between points $\mathrm{P}$ and $\mathrm{Q}(r)$ and the radius of the $\operatorname{arc}(q)$. On the other hand, when $Q$ is the central axis of the transmitter and point $O$ is the center of the receiver, the particle velocity on the arc with center $\mathrm{Q}$ and radius $q$ is equal, and the length of the arc can be expressed by the function of the radius of the receiver $(a)$, the distance between points $\mathrm{P}$ and $\mathrm{Q}(r)$ and the radius of the $\operatorname{arc}(q)$. For the integration on the transmitter and that on the receiver, the same subroutine can be used with different parameters.

\section{METHOD OF NUMERICAL INTEGRATION}

The numerical integration of the system is made by use of repeated trapezoidal rule. In the rule, the integration is approximated as follows:

$$
\begin{aligned}
W_{n} & =\int w d h \\
& =h / 2\left\{w_{0}+w_{2^{n}}+\left(w_{1}+w_{2}+\ldots+w_{i}+\ldots+w_{2^{n}-1}\right)\right\},
\end{aligned}
$$

where $n$ is an integer, $W_{n}$ the integral value, $h$ the quadruture coefficient denoting the subinterval of the integral and $w_{0}, w_{2}{ }^{n}$ and $w_{i}$ the initial value, the final value and the nodal value at the $i$-th point of the integrand, respectively. Increasing $n$ by one, the 


\section{T. IMAMURA: PRECISE COMPUTATION OF ULTRASONIC SYSTEMS}

number of subintervals is doubled, and the accuracy of the integral is gradually improved. Considering the accuracy and the required time of computation, the number $n$ which determines the subintervals is limited as

$$
n_{1} \leqq n \leqq n_{\mathrm{u}},
$$

and when

$$
\left|\dot{W}_{n}-\dot{W}_{n-1}\right|<0.005
$$

vals is stopped and the integral value is obtained.

In executing the calculation mentioned in Chapter 2, multiple integration is necessary. For example, double integration is achieved by calling a subprogram for integration while another subprogram is executing the integration.

\section{RESULTS}

As one measure, amplitude of the particle velocity is calculated for the response of the ultrasonic

Table 1 Response of the ultrasonic transmission system of a line transducer and a point one by numerical single integration $\left(z=5 a ;-a \leqq x^{\prime} \leqq a ; y^{\prime}=x=y=0\right)$.

\begin{tabular}{rccccccc}
\hline$n_{1}$ & \multicolumn{7}{c}{$a / \lambda$} \\
\cline { 2 - 7 } & 0.0 & 5.0 & 10.0 & 15.0 & 20.0 & 25.0 & 30.0 \\
\hline 1 & 0.00124 & 0.00490 & 0.39212 & 0.02953 & 0.78313 & 0.07786 & 1.17190 \\
2 & 0.00125 & 0.10144 & 0.27497 & 0.30233 & 0.02119 & 0.46067 & 0.86666 \\
3 & 0.00125 & 0.12040 & 0.14003 & 0.12357 & 0.38608 & 0.30216 & 0.41519 \\
4 & 0.00125 & 0.12434 & 0.12251 & 0.18640 & 0.19867 & 0.20504 & 0.30225 \\
5 & 0.00125 & 0.12529 & $\underline{0.11930}$ & 0.19602 & 0.18036 & 0.24126 & 0.23160 \\
6 & 0.00125 & 0.12552 & 0.11856 & $\underline{0.19822}$ & $\underline{0.17679}$ & 0.24787 & 0.22220 \\
7 & 0.00125 & 0.12558 & 0.11837 & 0.19876 & 0.17594 & $\underline{0.24943}$ & 0.22011 \\
8 & 0.00125 & 0.12559 & 0.11833 & 0.19890 & 0.17573 & 0.24981 & 0.21960 \\
9 & 0.00125 & 0.12560 & 0.11832 & 0.19893 & 0.17568 & 0.24991 & 0.21947 \\
10 & 0.00125 & 0.12560 & 0.11831 & 0.19894 & 0.17567 & 0.24993 & 0.21944 \\
11 & 0.00125 & 0.12560 & 0.11831 & 0.19894 & 0.17566 & 0.24994 & 0.21943 \\
\hline
\end{tabular}

Underlines indicate the data at $\left|\dot{W}_{n_{1}}-\dot{W}_{n_{1-1}}\right|<0.005$ when $4 \leqq n_{1} \leqq 11$.

Table 2 Response of the ultrasonic transmission system of a square transducer and a point one by numerical double integration $\left(z=5 a ;-a \leqq x^{\prime}, y^{\prime} \leqq a ; x=y=0\right)$.

\begin{tabular}{rccccccc}
\hline$n_{2}$ & \multicolumn{7}{c}{$a / \lambda$} \\
\cline { 2 - 7 } & 0.0 & 5.0 & 10.0 & 15.0 & 20.0 & 25.0 & 30.0 \\
\hline 1 & 0.02426 & 0.05805 & 2.32590 & 0.22970 & 3.44041 & 0.46336 & 4.27935 \\
2 & 0.02443 & 1.26458 & 1.63738 & 2.04265 & 0.07925 & 2.30489 & 3.14680 \\
3 & 0.02447 & 1.50652 & 0.83495 & 0.80794 & 1.70236 & 1.55959 & 1.52467 \\
4 & $\underline{0.02448}$ & 1.55700 & 0.73324 & 1.23683 & 0.87777 & 1.02056 & 1.10394 \\
5 & 0.02449 & 1.56911 & 0.71477 & 1.30295 & 0.79987 & 1.20904 & 0.85113 \\
6 & 0.02449 & $\underline{1.57210}$ & 0.71049 & 1.31806 & 0.78478 & 1.24347 & 0.81822 \\
7 & 0.02449 & 1.57285 & $\underline{0.70943}$ & $\underline{1.32176}$ & 0.78122 & 1.25157 & 0.81091 \\
8 & 0.02449 & 1.57304 & 0.70917 & 1.32268 & $\underline{0.78034}$ & $\underline{1.25357}$ & 0.80913 \\
9 & 0.02449 & 1.57308 & 0.70911 & 1.32291 & 0.78012 & 1.25407 & 0.80869 \\
10 & 0.02449 & 1.57309 & 0.70909 & 1.32297 & 0.78007 & 1.25419 & 0.80858 \\
11 & 0.02449 & 1.57310 & 0.70909 & 1.32298 & 0.78005 & 1.25422 & 0.80855 \\
\hline
\end{tabular}

Underlines indicate the data at $\left|\dot{W}_{n_{2}}-\dot{W}_{n_{2}-1}\right|<0.005$ when $4 \leqq n_{2} \leqq 11$. 
transmission systems. The value $a / \lambda$ is changed between 0 and 30, where $a$ is the half side length of a square transducer or the radius of a circular transducer, and $\lambda$ is the wavelength. The results are tabulated and are shown how integral converges according to the number of the nodal points.

Table 1 shows the response for the system of a line transducer and a point one by numerical single integration. The number of subintervals is $2^{n_{1}}$. Underlines indicate the data at $\left|\dot{W}_{n_{1}}-\dot{W}_{n_{1}-1}\right|<0.005$ when $4 \leqq n_{1} \leqq 11$. Increasing the value of $a / \lambda$, the integral converges more slowly. Table 2 shows the response for the system of a square transducer and a point one by numerical double integration. In calculating these data, the single integral is called as a subprogram where the boundary conditions are the same as the data with underlines in Table 1. Tables 3 and 4 show the response of the system of a square transducer and a line one, and that of a pair of square transducers. These are calculated by numerical triple and quadruple integrations respectively.

Table 5 shows the response for the system of a circular transducer and a point one, which is calculated by numerical single integration as mentioned in Chapter 2. The analytical results are also shown

Table 3 Response of the ultrasonic transmission system of a square transducer and a line one by numerical triple integration $\left(z=5 a ;-a \leqq x^{\prime}, y^{\prime}, x \leqq a ; y=0\right)$.

\begin{tabular}{cccccccc}
\hline & \multicolumn{7}{c}{$a / \lambda$} \\
$n_{3}$ & 0.0 & 5.0 & 10.0 & 15.0 & 20.0 & 25.0 & 30.0 \\
\cline { 2 - 8 } 1 & 0.02384 & 1.05846 & 0.54531 & 0.93082 & 0.60076 & 0.90856 & 0.63091 \\
2 & 0.02400 & 1.10200 & 0.75492 & 1.16400 & 0.81035 & 1.04899 & 0.73005 \\
3 & 0.02404 & 1.11905 & 0.76300 & 1.11325 & 0.81798 & 1.09602 & 0.80640 \\
4 & 0.02405 & 1.12118 & 0.77032 & 1.07311 & 0.82327 & 1.07544 & 0.84505 \\
5 & 0.02405 & 1.12178 & 0.77134 & 1.07628 & 0.82926 & 1.06329 & 0.85428 \\
6 & 0.02405 & 1.12193 & 0.77162 & 1.07727 & $\underline{0.82989}$ & $\underline{1.06363}$ & 0.85544 \\
7 & 0.02405 & 1.12197 & 0.77169 & 1.07743 & 0.83005 & 1.06382 & 0.85560 \\
8 & 0.02405 & 1.12198 & 0.77169 & 1.07753 & 0.83007 & 1.06403 & 0.85565 \\
9 & 0.02405 & 1.12198 & 0.77169 & 1.07754 & 0.83008 & 1.06404 & 0.85570 \\
10 & 0.02405 & 1.12198 & 0.77170 & 1.07754 & 0.83009 & 1.06403 & 0.85570 \\
11 & 0.02405 & 1.12198 & 0.77170 & 1.07754 & 0.83009 & 1.06403 & 0.85569 \\
\hline
\end{tabular}

Underlines indicate the data at $\left|\dot{W}_{n_{3}}-\dot{W}_{n_{3-1}}\right|<0.005$ when $4 \leqq n_{3} \leqq 11$.

Table 4 Response of the ultrasonic transmission system of a pair of square transducers by numerical quadruple integration $\left(z=5 a ;-a \leqq x^{\prime}, y^{\prime}, x, y \leqq a\right)$.

\begin{tabular}{cccccccc}
\hline & \multicolumn{7}{c}{$a / \lambda$} \\
\cline { 2 - 7 }$n_{4}$ & 0.0 & 5.0 & 10.0 & 15.0 & 20.0 & 25.0 & 30.0 \\
\hline 1 & 0.02342 & 0.74877 & 0.59011 & 0.75625 & 0.64136 & 0.76990 & 0.67186 \\
2 & 0.02357 & 0.78169 & 0.83074 & 0.94886 & 0.87534 & 0.88941 & 0.78045 \\
3 & $\underline{0.02361}$ & 0.79388 & 0.84050 & 0.90828 & 0.87883 & 0.93001 & 0.86106 \\
4 & 0.02362 & $\underline{0.79501}$ & 0.85167 & 0.87523 & 0.88578 & 0.91307 & 0.90220 \\
5 & 0.02362 & 0.79536 & 0.85275 & 0.87781 & 0.89359 & 0.90390 & 0.91119 \\
6 & 0.02362 & 0.79545 & 0.85303 & 0.87835 & 0.89436 & $\underline{0.90478}$ & 0.91242 \\
7 & 0.02362 & 0.79547 & 0.85310 & 0.87847 & 0.89453 & 0.90501 & 0.91267 \\
8 & 0.02362 & 0.79548 & 0.85312 & 0.87850 & 0.89457 & 0.90507 & 0.91273 \\
\hline
\end{tabular}

Underlines indicate the data at $\left|\dot{W}_{n_{4}}-\dot{W}_{n_{4}-1}\right|<0.005$ when $3 \leqq n_{4} \leqq 8$. 


\section{T. IMAMURA: PRECISE COMPUTATION OF ULTRASONIC SYSTEMS}

Table 5 Response of the ultrasonic transmission system of a circular transducer and a point one by numerical single integration $\left(z=5 a ; 0 \leqq r^{\prime} \leqq a ; r=0\right)$.

\begin{tabular}{cccccccc}
\hline$n_{1}$ & \multicolumn{7}{c}{$a / \lambda$} \\
\cline { 2 - 7 } & 0.0 & 5.0 & 10.0 & 15.0 & 20.0 & 25.0 & 30.0 \\
\hline 1 & 0.01928 & 1.21475 & 4.20901 & 8.64129 & 0.72961 & 13.70215 & 14.08309 \\
2 & 0.01938 & 1.78104 & 0.92658 & 1.15815 & 5.85740 & 10.46003 & 9.81630 \\
3 & 0.01941 & 1.93143 & 0.22651 & 1.50281 & 0.93915 & 0.51020 & 2.81514 \\
4 & 0.01942 & 1.96818 & 0.09068 & 1.86713 & 0.24532 & 1.65335 & 0.51218 \\
5 & 0.01942 & 1.97731 & 0.06844 & 1.95107 & 0.13716 & 1.89804 & 0.21594 \\
6 & 0.01942 & 1.97959 & 0.06494 & 1.97165 & 0.12527 & 1.95576 & 0.18622 \\
7 & 0.01942 & 1.98016 & $\underline{0.06423}$ & 1.97676 & 0.12381 & 1.96999 & 0.18402 \\
8 & 0.01942 & 1.98030 & 0.06407 & $\underline{1.97804}$ & $\underline{0.12355}$ & 1.97353 & 0.18382 \\
9 & 0.01942 & 1.98033 & 0.06403 & 1.97836 & 0.12349 & 1.97442 & 0.18379 \\
10 & 0.01942 & 1.98034 & 0.06402 & 1.97844 & 0.12347 & 1.97464 & 0.18378 \\
11 & 0.01942 & 1.98035 & 0.06401 & 1.97846 & 0.12347 & 1.97469 & 0.18378 \\
Analy. & 0.01942 & 1.08935 & 0.06401 & 1.97847 & 0.12347 & 1.97471 & 0.18378 \\
\hline
\end{tabular}

Underlines indicate the data at $\left|\dot{W}_{n_{1}}-\dot{W}_{n_{1-1}}\right|<0.005$ when $4 \leqq n_{1} \leqq 11$.

Table 6 Response of the ultrasonic transmission system of a pair of circular transducers by numerical double integration $\left(z=5 a ; 0 \leqq r^{\prime}, r \leqq a\right)$.

\begin{tabular}{cccccccc}
\hline$n_{2}$ & \multicolumn{10}{c}{$a / \lambda$} \\
\cline { 2 - 8 } & 0.0 & 5.0 & 10.0 & 15.0 & 20.0 & 25.0 & 30.0 \\
\hline 1 & 0.01864 & 0.62375 & 0.78630 & 0.85129 & 0.84354 & 0.78939 & 0.72522 \\
2 & 0.01876 & 0.77806 & 0.82379 & 0.85580 & 0.87315 & 0.92438 & 0.88230 \\
3 & 0.01879 & 0.80437 & 0.85322 & 0.86751 & 0.89037 & 0.92494 & 0.93358 \\
4 & 0.01879 & 0.81065 & 0.85739 & 0.87791 & 0.89347 & 0.90242 & 0.91229 \\
5 & 0.01880 & 0.81220 & 0.85835 & 0.88028 & $\underline{0.89458}$ & $\underline{0.90415}$ & $\underline{0.91215}$ \\
6 & 0.01880 & 0.81259 & 0.85859 & 0.88084 & 0.89485 & 0.90460 & 0.91211 \\
7 & 0.01880 & 0.81269 & 0.85862 & 0.88097 & 0.89490 & 0.90471 & 0.91213 \\
8 & 0.01880 & 0.81271 & 0.85864 & 0.88100 & 0.89492 & 0.90474 & 0.91212
\end{tabular}

Underlines indicate the data at $\left|\dot{W}_{n_{2}}-\dot{W}_{n_{2}-1}\right|<0.005$ when $3 \leqq n_{2} \leqq 8$.

at the bottom of the table. Table 6 shows the response of the system of a pair of circular transducers by numerical double integration. Table 7 shows the amplitude of the velocity potential multiplied by $2 \pi / \lambda$ of the ultrasonic transmission system of a pair of square transducers by numerical quadruple integration, which corresponds to the data of Table 4. Comparing these tables, the convergency is found to be the same and the calculated results differ a little.

Figures 3 and 4 show how is the convergency of the numerical integrations. Figure 3 shows the results for square transducers and Fig. 4 shows the results for circular transducers. In the case of circular transducers, the convergency of the double integral shows a little disorder with respect to the value of $a / \lambda$. This seems because the analytical decrease of numerical integrity has some effect on the convergency of the integral. Figures 5 and 6 show the responses of a pair of square transducers and those of a pair of circular ones. From these figures, it can be noted that the difference of the transducers' shape does not affect much to their response. In the case where the distance of the transducers is $5 a$, and the 
Table 7 Amplitude of the velocity potential multiplied by $2 \pi / \lambda$ of the ultrasonic transmission system of a pair of square transducers by numerical quadruple integration $(z=5 a$, $\left.-a \leqq x^{\prime}, y^{\prime}, x, y \leqq a\right)$.

\begin{tabular}{cccccccc}
\hline & \multicolumn{7}{c}{$a / \lambda$} \\
\cline { 2 - 7 }$n_{4}$ & 0.0 & 5.0 & 10.0 & 15.0 & 20.0 & 25.0 & 30.0 \\
\hline 1 & 0.0 & 0.74769 & 0.58664 & 0.75651 & 0.63971 & 0.77104 & 0.67137 \\
2 & 0.0 & 0.78081 & 0.83028 & 0.95048 & 0.87601 & 0.89078 & 0.78022 \\
3 & 0.0 & 0.79350 & 0.83981 & 0.90987 & 0.87866 & 0.93095 & 0.86042 \\
4 & 0.0 & $\underline{0.79450}$ & 0.85162 & 0.87531 & 0.88560 & 0.91373 & 0.90201 \\
5 & 0.0 & 0.79481 & $\underline{0.85271}$ & $\underline{0.87798}$ & 0.89391 & 0.90384 & 0.91122 \\
6 & 0.0 & 0.79491 & 0.85299 & 0.87854 & $\underline{0.89474}$ & $\underline{0.90479}$ & 0.91251 \\
7 & 0.0 & 0.79494 & 0.85306 & 0.87866 & 0.89491 & 0.90502 & 0.91277 \\
8 & 0.0 & 0.79496 & 0.85307 & 0.87869 & 0.89494 & 0.90509 & 0.91283 \\
\hline
\end{tabular}

Underlines indicate the data at $\left|\dot{W}_{n_{4}}-\dot{W}_{n_{4}-1}\right|<0.005$ when $3 \leqq n_{4} \leqq 8$.

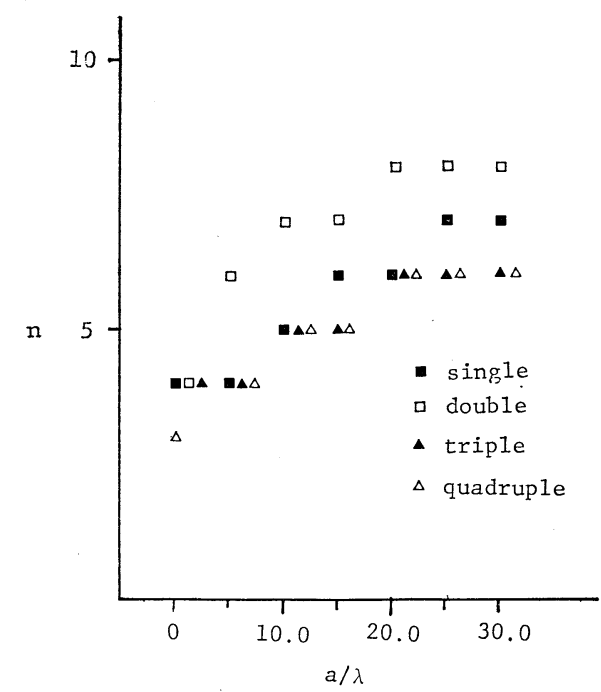

Fig. 3 Convergency of the numerical integration for square transducers $(z=5 a)$.

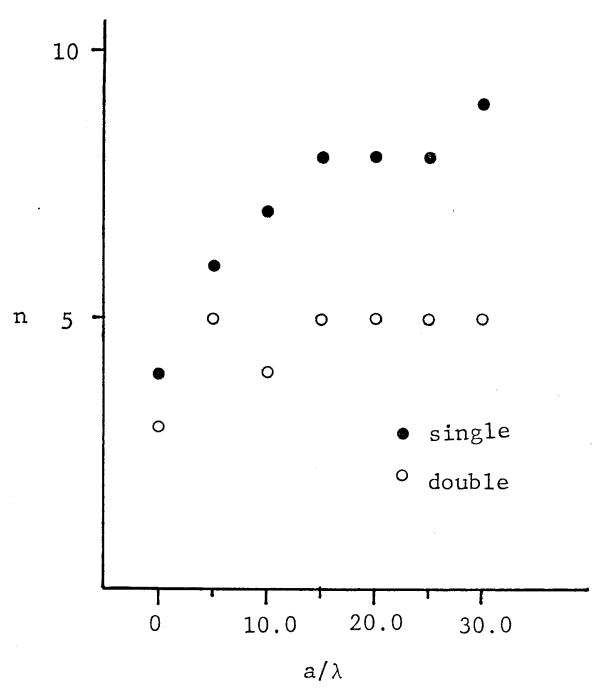

Fig. 4 Convergency of the numerical integration for circular transducers $(z=5 a)$. values of $a / \lambda$ are $5,10,15,20,25$ and 30 , the differences become $2.2 \%, 0.6 \%, 0.3 \%, 0.03 \%, 0.07 \%$ and $0.03 \%$, respectively.

\section{CONCLUSION}

The response of square transducers and that of circular ones are calculated precisely and are tabulated. The former is obtained by numerical quadruple integration. On the other hand, the latter is obtained by numerical double integration, and this shows a little irregularity of the convergency of the integral.
The particle velocity and velocity potential multiplied by $2 \pi / \lambda$ show little difference on the convergency of the integral. The calculated results of them show a little difference. The response of the system of a pair of square transducers and that of circular ones show a similar tendency with respect to $a / \lambda$ and the distance between transducers, but a certain difference is found by the precise investigation.

\section{ACKNOWLEDGEMENTS}

The author is grateful to Mr. H. Imai and Dr. S. 


\section{T. IMAMURA: PRECISE COMPUTATION OF ULTRASONIC SYSTEMS}

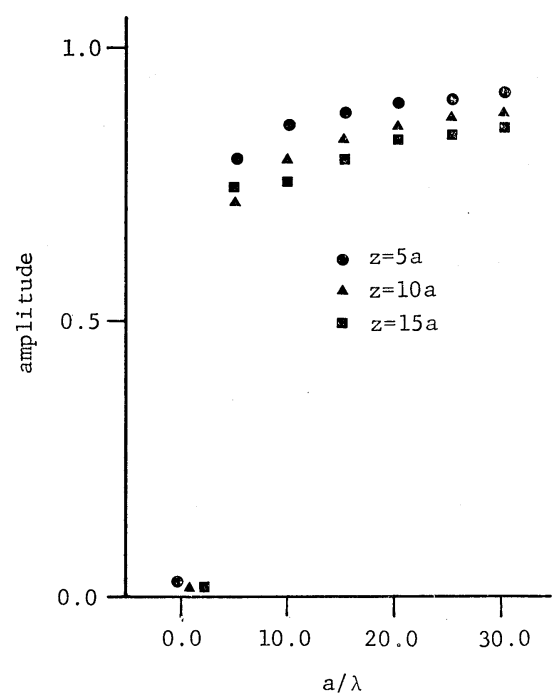

Fig. 5 Response of the system of a pair of square transducers.

Nagai of NRLM for their comments and discussions.

\section{REFERENCES}

1) H. Seki, A. Granato, and R. Truell, "Diffraction effects in the ultrasonic field of a piston source and their importance in the accurate measurement of attenuation," J. Acoust. Soc. Am. 28, 230-238 (1956).

2) Y. Fujii and K. Yamada, "Acoustic response of a rectangular receiver to a rectangular source," $\mathbf{J}$. Acoust. Soc. Am. 40, 249-251 (1966).

3) K. Yamada and Y. Fujii, "Acoustic response of a circular receiver to a circular source of different radius," J. Acoust. Soc. Am. 40, 1193-1194 (1966).

4) J. Zemanek, "Beam behavior within the nearfield of a vibrating piston," J. Acoust. Soc. Am. 49, 181191 (1971).

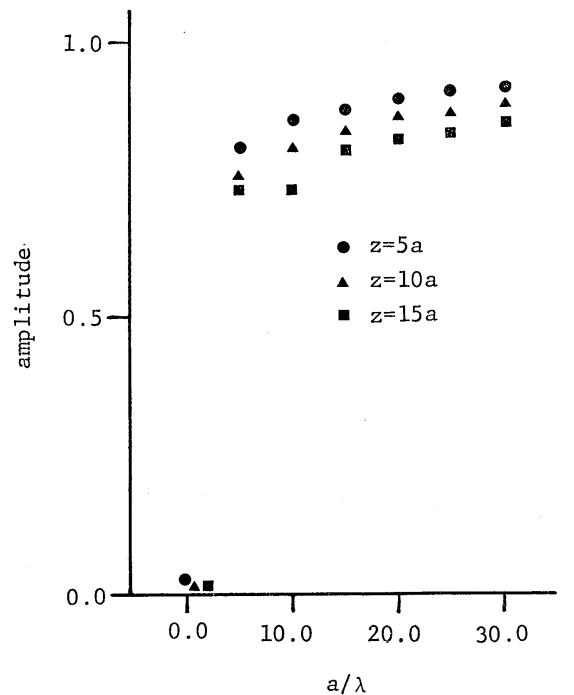

Fig. 6 Response of the system of a pair of circular transducers.

5) K. Harumi, F. Suzuki, and Y. Sato, "Computer simulation of the nearfield for elastic wave in a solid half-space," J. Acoust. Soc. Am. 53, 660-664 (1973).

6) T. Imamura and K. Iizuka, "Effect of beam divergence on ultrasonic attenuation measurement," Proc. 21st Jpn. Nat. Cong. Appl. Mech. (Univ. of Tokyo Press, Tokyo, 1973), pp. 167-176.

7) S. Ohtsuki, "Calculation method for the nearfield of a sound source with ring function," J. Acoust. Soc. Jpn. 30, 76-81 (1974) (in Japanese).

8) A. S. Khimunin, "Ultrasonic propagation parameter measurements incorporating exact diffraction corrections," Acustica 39, 87-95 (1978).

9) T. Imamura, "Numerical analysis of ultrasonic transmission system of rectangular transducers; Effect of translation, rotation and tilt," Bull. NRLM 33, 205-211 (1984). 